\title{
Dimension-Specific Efficiency Measurement Using Data Envelopment Analysis
}

\author{
Hongjun Zhang, Youliang Zhang, and Rui Zhang \\ Institute of Command and Information Systems, PLA University of Science and Technology, Nanjing 210007, China \\ Correspondence should be addressed to Youliang Zhang; zhyldehao@yeah.net
}

Received 26 October 2014; Revised 6 December 2014; Accepted 6 December 2014; Published 25 December 2014

Academic Editor: Wei-Chiang Hong

Copyright (C) 2014 Hongjun Zhang et al. This is an open access article distributed under the Creative Commons Attribution License, which permits unrestricted use, distribution, and reproduction in any medium, provided the original work is properly cited.

\begin{abstract}
Data envelopment analysis (DEA) is a powerful tool for evaluating and improving the performance of a set of decision-making units (DMUs). Empirically, there are usually many DMUs exhibiting "efficient" status in multi-input multioutput situations. However, it is not appropriate to assert that all efficient DMUs have equivalent performances. Actually, a DMU can be evaluated to be efficient as long as it performs best in a single dimension. This paper argues that an efficient DMU of a particular input-output proportion has its own specialty and may also perform poorly in some dimensions. Two DEA-based approaches are proposed to measure the dimension-specific efficiency of DMUs. One is measuring efficiency in multiplier-form by further processing the original multiplier DEA model. The other is calculating efficiency in envelopment-form by comparing with an ideal DMU. The proposed approaches are applied to 26 supermarkets in the city of Nanjing, China, which have provided new insights on efficiency for the managers.
\end{abstract}

\section{Introduction}

Data envelopment analysis (DEA) [1] is a mathematical programming method for evaluating the relative efficiency of decision-making units (DMUs) with multiple outputs and multiple inputs. By using DEA, a single index (namely, efficiency score) can be obtained from the ratio of weighted outputs to weighted inputs, as an assessment of a DMU's overall performance.

Introduced by Charnes et al. in 1978 [1], DEA has been recognized as an excellent and easily used methodology for modeling operational processes for performance evaluations [2]. It has been widely applied in many application areas [3], such as banks $[4,5]$, agriculture $[6,7]$, health care $[8,9]$, education $[10,11]$, and transportation $[12,13]$.

There are several reasons why DEA can be successfully applied $[3,14]$. First, it is a nonparametric technique that does not require any underlying assumptions on the production function defining the relationship between inputs and outputs. Second, it can distinguish between efficient and inefficient DMUs. Third, multiple inputs and outputs can be considered simultaneously. Finally, it can pinpoint the sources and the amount of deficiency for each of the inefficient DMUs and provide proper benchmarking information for them to make improvement.

However, in the extensive applications, most attentions have been paid on the evaluation of overall performances, whereas no literature focuses on how a DMU performs in an individual input/output dimension. Since a single index is used to evaluate a DMU's performance, efficient DMUs, with a maximum efficiency score of unity, are usually considered perfect with no room for improvement. Actually, it is not always the case. Empirically, there are usually multiple units exhibiting "efficient" status in multi-input and multioutput situations [15]. In this study, we argue that efficient DMUs of different input-output proportions have different production specialties and may also perform poorly in some aspects. With the flexibility of weights selection, a DMU can achieve an "efficient" status as long as it performs best in very few dimensions, no matter how poor it is in other dimensions. A larger weight can be assigned to its best-practice dimensions and an infinitesimal value (even zero, if allowed) to its weak points.

Furthermore, as we all know, DEA is sensitive to variable selection [16]. Measuring efficiency in each dimension, to some extent, can reduce the sensitivity. 
Therefore, it is of great significance to measure dimension-specific efficiency of DMUs. A simple example can be used to illustrate this problem. Suppose that there are two supermarkets A and B in the same metropolitan area, each using two inputs (promotion expenses and number of employees) to produce the same amount of outputs. A spends less in promotion but has more employees than $\mathrm{B}$. Then both $\mathrm{A}$ and $\mathrm{B}$ will be evaluated as efficient by the original DEA model. After investigation we find that $\mathrm{A}$ is good at promotion, whereas B is good at staff management. Therefore, more information can be obtained by measuring efficiency in each dimension.

This paper proposes two approaches for measuring the dimension-specific efficiency of DMUs. One is to measure efficiency in a multiplier-form by further processing the original multiplier DEA model. The other is to calculate efficiency in an envelopment-form by comparing with an ideal DMU. The two approaches evolve from the "multiplier side" and the "envelopment side" of the original DEA model, respectively. Different ideas are used for the definition of dimension-specific efficiency, so the new models are not dual problems. Therefore, efficiency scores calculated by the two approaches will not be necessarily the same.

The remainder of the paper is organized as follows. Sections 2 and 3 present the two proposed approaches, respectively. Both approaches are demonstrated by the same simple numerical example. Section 4 applies the two approaches to a real world data set consisting of 26 supermarkets. Finally, Section 5 summarizes our work.

\section{Multiplier Models for Dimension-Specific Efficiency Measurement: Approach 1}

2.1. Multiplier CCR Model. Assume there are $n$ DMUs under evaluation. Each $\mathrm{DMU}_{j}(j=1,2, \ldots, n)$ consumes $m$ inputs $x_{i j}(i=1,2, \ldots, m)$ to produce $s$ outputs $y_{r j}(r=1, \ldots, s)$. The efficiency score for any evaluated $\mathrm{DMU}_{p}$ is defined as the maximal ratio of its total weighted output to its total weighted input, under the constraints that the efficiency for each DMU cannot exceed unity. The mathematical programming problem can thus be stated as the following multiplier CCR model [1]:

$$
\begin{aligned}
& z_{p}=\max \frac{\sum_{r=1}^{s} u_{r} y_{r p}}{\sum_{i=1}^{m} v_{i} x_{i p}} \\
& \text { subject to } \quad \frac{\sum_{r=1}^{s} u_{r} y_{r j}}{\sum_{i=1}^{m} v_{i} x_{i j}} \leq 1, \quad j=1, \ldots, n, \\
& \\
& v_{i}, u_{r} \geq 0, \quad \forall i, r,
\end{aligned}
$$

where $u_{r}$ and $v_{i}$ are multipliers to the outputs and inputs, respectively.

This fractional programming problem can be transformed into a linear programming problem using the Charnes-Cooper transformation [1] as

$$
z_{p}=\max \sum_{r=1}^{s} \mu_{r} y_{r p}
$$

$$
\begin{array}{ll}
\text { subject to } & \sum_{r=1}^{s} \omega_{i} x_{i p}=1, \\
& \sum_{r=1}^{s} \mu_{r} y_{r j}-\sum_{r=1}^{s} \omega_{i} x_{i j} \leq 0, \quad j=1, \ldots, n, \\
& \omega_{i}, \mu_{r} \geq 0, \quad \forall i, r .
\end{array}
$$

Model (2) is input-oriented and under constant returnsto-scale (CRS) assumption. The performance of $\mathrm{DMU}_{p}$ is usually considered to be CCR DEA efficient if $z_{p}=1$ and CCR DEA inefficient if $z_{p}<1$.

2.2. Evaluation. A characteristic of the original DEA model is that it allows each DMU to measure the efficiency using its favorable weights in order to calculate its maximum efficiency score [17]. With the flexibility of weights selection, a DMU would assign a larger multiplier (weight) to the dimension where it has a better performance. That is, a smaller input or a bigger output, compared with other DMUs, would be assigned a larger multiplier. In contrast, a bigger input or a smaller output would be assigned a smaller multiplier.

Denote $I_{i}(i=1, \ldots, m)$ as the $i$ th input dimension. In the optimal solution of model (2) we can find that if $\mathrm{DMU}_{p}$ performs better in input dimension $I_{o}, x_{o p}$ would then obtain a greater ratio of its weighted value to $\mathrm{DMU}_{p}$ 's total weighted input; that is, $\omega_{o} x_{o p} / \sum_{i=1}^{m} \omega_{i} x_{i p}$. Since the constraint $\sum_{i=1}^{m} \omega_{i} x_{i p}=1$ is set in model (2), the ratio can be reduced to (equals to) $\omega_{o} x_{o p}$.

However, sometimes model (2) encounters the existence of alternative weights, especially in the case of the extreme efficient units [2]. For example, if $\mathrm{DMU}_{p}$ has the most excellent performance both in dimension $I_{M}$ and in dimension $I_{N}$, either $\omega_{M} x_{M p}=1$ or $\omega_{N} x_{N p}=1$ would satisfy the constraints in model (2), without affecting $\mathrm{DMU}_{p}$ 's relative efficiency. To solve this problem, a two-stage process is developed as follows.

Stage 1. Solve model (2) to calculate efficiency score $z_{p}$ of $\mathrm{DMU}_{p}$.

Stage 2. Solve the following model to get the maximal value of $e_{o p}^{I}$ :

$$
\begin{array}{ll}
e_{o p}^{I}=\max & \omega_{o} x_{o p} \\
\text { subject to } & \sum_{r=1}^{s} \omega_{i} x_{i p}=1, \\
& \sum_{r=1}^{s} \mu_{r} y_{r p}=z_{p}, \\
& \sum_{r=1}^{s} \mu_{r} y_{r j}-\sum_{r=1}^{s} \omega_{i} x_{i j} \leq 0, \quad j=1, \ldots, n, \\
& \omega_{i}, \mu_{r} \geq 0, \quad \forall i, r,
\end{array}
$$


where $0 \leq e_{o p}^{I} \leq 1$. The product of $e_{o p}^{I}$ and $z_{p}$ could be interpreted as an index reflecting the utilization efficiency of input $x_{o p}$ by $\mathrm{DMU}_{p}$.

The developments above can lead to the following definition to evaluate $\mathrm{DMU}_{p}$ 's performance in input dimension $I_{o}$.

Definition 1 (input dim-efficiency score, by Approach 1, input-oriented, under CRS assumption). One defines the input dim-efficiency score of $\mathrm{DMU}_{p}$ in dimension $I_{o}$ as the product of its maximal weighted value and its CCR efficiency score; that is, $\rho_{o p}^{I}=e_{o p}^{I} z_{p}$.

By the following definition we can distinguish DMUs' performance in input dimension $I_{o}$ into two separate classes, dim-efficient and dim-inefficient.

Definition 2 (input dim-efficiency, by Approach 1). If $\rho_{o p}^{I}=1$, then $\mathrm{DMU}_{p}$ is dim-efficient in input dimension $I_{o}$; if $\rho_{o p}^{I}<1$, then $\mathrm{DMU}_{p}$ is dim-inefficient in input dimension $I_{o}$.

Alternately, we turn to performance evaluation in output dimensions. Denote $O_{r}(r=1, \ldots, s)$ as the $r$ th output dimension. Similar to input dimension, we can use a twostage process to measure the efficiency on output $O_{o}$. The first stage is the same, whereas the model in the second stage is different, which is now as follows, aiming to maximize the weighted value of $y_{o p}$ to $\mathrm{DMU}_{p}$ 's weighted sum of outputs:

$$
\begin{array}{ll}
e_{o p}^{O}=\max & \frac{\mu_{o} y_{o p}}{z_{p}} \\
\text { subject to } & \sum_{r=1}^{s} \omega_{i} x_{i p}=1, \\
& \sum_{r=1}^{s} \mu_{r} y_{r p}=z_{p}, \\
& \sum_{r=1}^{s} \mu_{r} y_{r j}-\sum_{r=1}^{s} \omega_{i} x_{i j} \leq 0, \quad j=1, \ldots, n, \\
& \omega_{i}, \mu_{r} \geq 0, \quad \forall i, r .
\end{array}
$$

Denote $\mu_{r}^{*}$ and $\omega_{i}^{*}$ as the optimal solution of model (4); then $\mathrm{DMU}_{p}$ 's performance in output dimension $\mathrm{O}_{o}$ can be assessed by the following definition.

Definition 3 (output dim-efficiency score, by Approach 1, input-oriented, under CRS assumption). One defines the output dim-efficiency score of $\mathrm{DMU}_{p}$ in dimension $O_{o}$ as $\rho_{o p}^{O}=$ $e_{o p}^{\mathrm{O}} \times z_{p}=\left(\mu_{o}^{*} y_{o p} / z_{p}\right) z_{p}=\mu_{o}^{*} y_{o p}$.

We can also distinguish between dim-efficient and diminefficient DMUs in output dimension $O_{o}$ by the following definition.

Definition 4 (output dim-efficiency, by Approach 1). If $\rho_{o p}^{O}=$ 1 , then $\mathrm{DMU}_{p}$ is dim-efficient in output dimension $O_{o}$; if $\rho_{o p}^{O}<1$, then $\mathrm{DMU}_{p}$ is dim-inefficient in output dimension $\mathrm{O}_{o}$.

Now we have the following propositions.

Proposition 5. $\rho_{o p}^{I}$ and $\rho_{o p}^{O}$ are unit-invariant; that is, they are independent of the units in which the inputs and outputs are measured.

It holds because models in calculation process, (2), (3), and (4), are all units-invariant.

Proposition 6. $\rho_{o p}^{I}$ and $\rho_{o p}^{O}$ are scale-invariant; that is, they are independent of the differences among DMUs' production scales but depend only on input and output proportions, respectively.

It holds since the evaluation result is based on ratio scores and not on the input/output numerical values.

Proposition 7. Being CCR DEA efficient is a necessary condition for a DMU to be dim-efficient.

Proof. Suppose $\mathrm{DMU}_{p}$ is dim-efficient in input dimension $I_{o}$; then we have $\rho_{o p}^{I}=e_{o p}^{I} z_{p}=1$. Since $0 \leq e_{o p}^{I} \leq 1$, so $z_{p}=1$, which indicates $\mathrm{DMU}_{p}$ is CCR DEA efficient.

Similarly, suppose $\mathrm{DMU}_{p}$ is dim-efficient in output dimension $O_{o}$; then we have $\rho_{o p}^{O}=e_{o p}^{O} z_{p}=1$. Since $0 \leq$ $e_{o p}^{O} \leq 1$, so $z_{p}=1$, which also indicates the CCR efficiency of $\mathrm{DMU}_{p}$.

A DMU's being dim-efficient means that it has the most excellent performance in this dimension and should serve as a role model for dim-inefficient DMUs to imitate. On the contrary, a dim-efficient DMU fails to achieve a score of 1.0 because of the presence of another DMU that receives a higher ratio for the same set of weights.

Now we use a simple numerical example to illustrate the above definitions. Table 1 presents a data set for 6 DMUs with two inputs and a single output, along with their CCR efficiency scores from model (2) in the last row. In parenthesis are their dim-efficiency scores in the associated dimensions.

Table 1 tells us that even an extreme efficient DMU can be evaluated dim-inefficient in some dimensions, see DMU3, for example, which is CCR DEA efficient but only gets a dimefficiency score of 0.667 in Input 1 . An extreme efficient DMU can have its own production specialty. For instance, DMU3 is excellent at the utilization of Input 2, whereas DMU5 performs best in Input 1 .

\section{Envelopment Models for Dimension-Specific Efficiency Measurement: Approach 2}

3.1. Evaluation. In this section, we turn to the dual side of the DEA model, envelopment model, to measure DMUs' efficiency in each dimension. Firstly, we consider the dual model 
TABLE 1: Input-output data for 6 DMUs and their dim-efficiency scores.

\begin{tabular}{lcccccc}
\hline DMU & 1 & 2 & 3 & 4 & 5 & 6 \\
\hline Input 1 & $2(0.490)$ & $3(0.368)$ & $4(0.667)$ & $2(0.667)$ & $1(1)$ & $5(0.005)$ \\
Input 2 & $3(0.368)$ & $2(0.490)$ & $1(1)$ & $2(0.667)$ & $4(0.667)$ & $1(1)$ \\
Output 1 & $1(0.857)$ & $1(0.857)$ & $1(1)$ & $1(1)$ & $1(1)$ & $1(1)$ \\
CCR efficiency score & 0.857 & 0.857 & 1 & 1 & 1 & 1 \\
\hline
\end{tabular}

of (3), which is stated as the following linear programing problem:

$$
\begin{array}{ll}
\min & \varphi_{1}-z_{p} \varphi_{2} \\
\text { subject to } & \sum_{j=1}^{n} \lambda_{j} x_{o j} \leq\left(\varphi_{1}-1\right) x_{o p}, \\
& \sum_{j=1}^{n} \lambda_{j} x_{i j} \leq \varphi_{1} x_{i p}, \quad i=1, \ldots, m, \\
& \sum_{j=1}^{n} \lambda_{j} y_{r j} \geq \varphi_{2} y_{r p}, \quad r=1, \ldots, s, \\
& \lambda_{j} \geq 0 \quad j=1, \ldots, n, \\
& \varphi_{1}, \varphi_{2} \quad \text { free. }
\end{array}
$$

Model (5) can also be used for dimensional efficiency measurement, which will get an optimal solution equal to its primal dual model (3).

However, (5) is not an easily understood measure. Therefore, we extend the existing DEA model and construct an ideal $D M U$ for both input side and output side.

Definition 8 (ideal DMU, input side). Take DMU, 's outputs as the output isoquant, then one can construct an ideal DMU with outputs equal to $\mathrm{DMU}_{p}$ 's and inputs derived by varying "o" in model (6). Then the ideal DMU can be expressed as $\mathrm{DMU}_{\text {ideal }}^{I}=\left(\mathbf{x}_{\text {ideal }}, \mathbf{y}_{p}\right), \mathbf{x}_{\text {ideal }}=\left\{x_{1}^{\text {ideal }}, \ldots, x_{m}^{\text {ideal }}\right\}$, and $\mathbf{y}_{p}=$ $\left\{y_{1 p}, \ldots, y_{s p}\right\}$. Consider

$$
\begin{aligned}
& x_{o}^{\text {ideal }}=\min x_{o} \\
& \text { subject to } \sum_{j=1}^{n} x_{o j} \lambda_{j} \leq x_{o}, \\
& \qquad \sum_{j=1}^{n} y_{r j} \lambda_{j} \geq y_{r p}, \quad r=1, \ldots, s, \\
& \lambda_{j} \geq 0, \quad j=1, \ldots, n .
\end{aligned}
$$

The purpose of (6) is to find a linear combination of the observed DMUs, such that the minimum input in the input dimension concerned can be identified, with outputs no less than $\mathrm{DMU}_{p}$ 's. Figure 1 depicts the ideal DMU $P_{\text {ideal }}$ for the data in Table 1 with unit output isoquant.

Then, dimension-specific efficiency can be measured by comparing $\mathrm{DMU}_{p}$ with the ideal DMU. In Figure 1, for

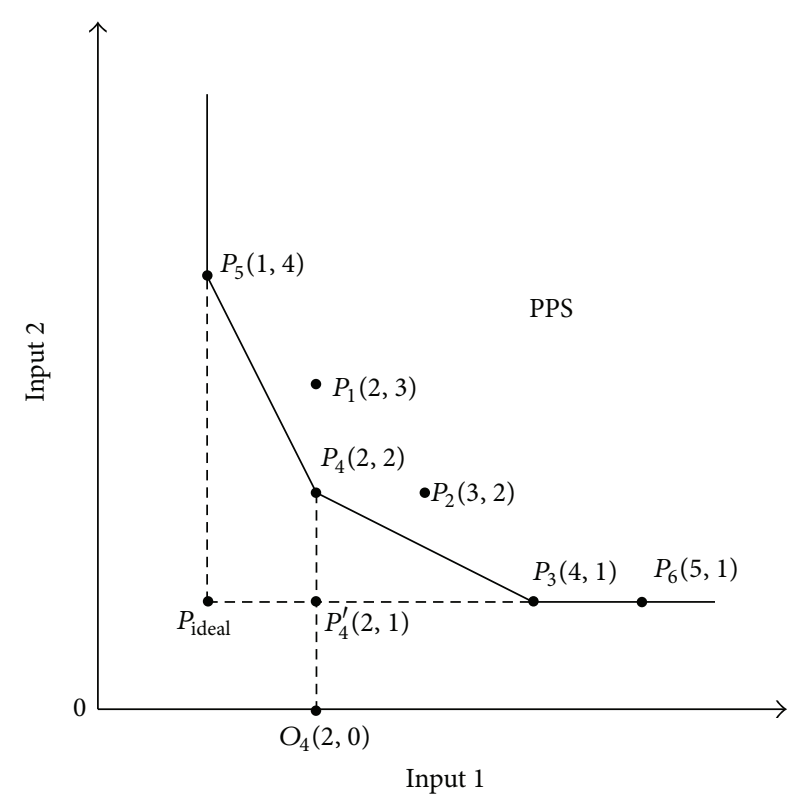

FIGURE 1: Ideal DMU with unit output isoquant.

example, $P_{4}$ 's dim-efficiency score in Input 2 can be calculated as $O_{4} P_{4}^{\prime} / O_{4} P_{4}$; that is, $\theta_{2 p}^{I}=x_{2}^{\text {ideal }} / x_{2 p}=0.5$.

So the following model can be used to calculate $\mathrm{DMU}_{p}$ 's dim-efficiency score in input dimension $I_{o}$ :

$$
\begin{aligned}
\theta_{o p}^{I}=\min \frac{x_{o}}{x_{o p}} & \\
\text { subject to } & \sum_{j=1}^{n} x_{o j} \lambda_{j} \leq x_{o}, \\
& \sum_{j=1}^{n} y_{r j} \lambda_{j} \geq y_{r p}, \quad r=1, \ldots, s, \\
& \lambda_{j} \geq 0, \quad j=1, \ldots, n,
\end{aligned}
$$

where $x_{o}$ and $\lambda_{j}(j=1, \ldots, n)$ are unknown variables.

By making the change of variables $\varphi=x_{o} / x_{o p}$ and $x_{o}=$ $\varphi x_{o p}$, model (7) can be converted to the following problem:

$$
\begin{aligned}
& \theta_{o p}^{I}=\min \varphi \\
& \text { subject to } \sum_{j=1}^{n} x_{o j} \lambda_{j} \leq \varphi x_{o p}
\end{aligned}
$$




$$
\begin{aligned}
& \sum_{j=1}^{n} y_{r j} \lambda_{j} \geq y_{r p}, \quad r=1, \ldots, s, \\
& \lambda_{j} \geq 0, \quad j=1, \ldots, n, \\
& \varphi \quad \text { free. }
\end{aligned}
$$

The above developments now lead to the following definition.

Definition 9 (input dim-efficiency score, by Approach 2, under CRS assumption). The input dim-efficiency score of $\mathrm{DMU}_{p}$ in dimension $I_{o}$ is defined as $\theta_{o p}^{I}$ calculated by model (8).

Alternately, in a similar manner, an ideal DMU on the output side can be obtained by the following definition.

Definition 10 (ideal DMU, output side). Given a certain amount of resources (suppose the same as $\mathrm{DMU}_{p}$ 's inputs), an ideal DMU can be constructed by solving model (9). One can show the ideal DMU with $\mathrm{DMU}_{\text {ideal }}^{O}=\left(\mathbf{x}_{p}, \mathbf{y}_{\text {ideal }}\right), \mathbf{x}_{p}=$ $\left\{x_{1 p}, \ldots, x_{m p}\right\}$, and $\mathbf{y}_{\text {ideal }}=\left\{y_{1}^{\text {ideal }}, \ldots, y_{s}^{\text {ideal }}\right\}$. Consider

$$
\begin{aligned}
& y_{o}^{\text {ideal }}=\max y_{o} \\
& \text { subject to } \sum_{j=1}^{n} x_{i j} \lambda_{j} \leq x_{i p}, \quad i=1, \ldots, m, \\
& \qquad \sum_{j=1}^{n} y_{o j} \lambda_{j} \geq y_{o} \\
& \lambda_{j} \geq 0, \quad j=1, \ldots, n .
\end{aligned}
$$

From the definitions we can see that ideal DMUs on both input side and output side would change for each different DMU, which are determined by the input/output isoquants.

Compare $\mathrm{DMU}_{p}$ with the ideal DMU using linear programing problem

$$
\begin{aligned}
& \varphi^{*}=\max \varphi \\
& \text { subject to } \sum_{j=1}^{n} x_{i j} \lambda_{j} \leq x_{i p}, \quad i=1, \ldots, m, \\
& \\
& \sum_{j=1}^{n} y_{o j} \lambda_{j} \geq \varphi y_{o p}, \\
& \lambda_{j} \geq 0, \quad j=1, \ldots, n, \\
& \varphi \quad \text { free. }
\end{aligned}
$$

Definition 11 (output dim-efficiency score, by Approach 2, under CRS assumption). The output dim-efficiency score of $\mathrm{DMU}_{p}$ in dimension $O_{o}$ is defined as $\theta_{o p}^{O}=\left(\varphi^{*}\right)^{-1}$, where $\varphi^{*}$ is calculated by model (10).
TABLE 2: Dimensional efficiencies derived by Approach 2.

\begin{tabular}{lcccccc}
\hline DMU & 1 & 2 & 3 & 4 & 5 & 6 \\
\hline$\theta_{1 j}^{I}$ & 0.5 & 0.333 & 0.25 & 0.5 & 1 & 0.2 \\
$\theta_{2 j}^{I}$ & 0.333 & 0.5 & 1 & 0.5 & 0.25 & 1 \\
$\theta_{1 j}^{O}$ & 0.857 & 0.857 & 1 & 1 & 1 & 1 \\
\hline
\end{tabular}

Now we can distinguish dim-efficient DMUs from diminefficient ones by Definition 12, on both input side and output side.

Definition 12 (dim-efficiency, by Approach 2). If $\theta_{o p}=1$ (here $\theta_{o p}$ refers to either an input or an output dim-efficiency score), then $\mathrm{DMU}_{p}$ is dim-efficient in the associated dimension; if $\theta_{o p}<1$, then $\mathrm{DMU}_{p}$ is dim-inefficient in the associated dimension.

Approach 2 also has the following properties.

Proposition 13. $\theta_{o p}^{I}$ and $\theta_{o p}^{O}$ are units-invariant and scalesinvariant.

Table 2 shows the dim-efficiency scores of DMUs in Table 1, derived by Approach 2, which also implies that efficient DMUs have their own specialties, as well as weak points in some dimensions. Dim-efficiencies scores in Tables 1 and 2 are not the same, because the calculation models are not dual problems which are formed using different ideas.

3.2. Benchmarking. This section provides benchmarking information for dim-inefficient DMUs to make improvement. Conventional benchmarking methods suggest that DMUs in the reference-set should be learned from an overall perspective, without focusing on specific dimensions. However, there could be two shortcomings: one is that DMUs in the reference-set do not necessarily perform better than the evaluated DMU in every dimension, which may even lead to retrogression; the other is that setting a single target at each benchmark learning step may slow substantially the pace of improvement or cannot fully tap the potential of the evaluated DMU.

Therefore, we identify reference-set for the evaluated DMU in each dimension, which not only has a specific focus but also can speed up the improvement process.

Let the optimal solution of models (8) and (10) be $\lambda^{*}$. Then, based on $\lambda^{*}$, we define the reference-set to $\mathrm{DMU}_{p}$ as follows.

Definition 14 (reference-set). The set of indices corresponding to positive $\lambda_{j}^{*} s$ is called the reference-set to $\mathrm{DMU}_{p}$ in the corresponding dimension.

In the occurrence of multiple optimal solutions, the reference-set is not unique. We can choose any one for our purpose [18].

The reference-set $R_{o}$ is

$$
R_{o}=\left\{j \mid \lambda_{j}^{*}>0\right\}, \quad(j \in\{1, \ldots, n\}) .
$$


TABLE 3: Reference-set for DMUs in Table 1.

\begin{tabular}{lcccccc}
\hline DMU & 1 & 2 & 3 & 4 & 5 & 6 \\
\hline Input 1 & 5 & 5 & 5 & 5 & & 5 \\
Input 2 & 3 & 3 & & 3 & 3 & 3 \\
Output 1 & $4(71 \%)$, & $3(29 \%)$, & & & & 3 \\
& $5(29 \%)$ & $4(71 \%)$ & & & & \\
\hline
\end{tabular}

Using $R_{o}$, we can express the target of $\mathrm{DMU}_{p}$ by $\mathrm{DMP}_{p}^{\prime}=$ $\left(\mathbf{x}_{p}^{\prime}, \mathbf{y}_{p}^{\prime}\right)$, where (input-oriented target)

$$
\begin{gathered}
\mathbf{x}_{p}^{\prime}=\left\{\sum_{j \in R_{o}} x_{i j} \lambda_{j}^{*} \mid i=1, \ldots, m\right\}, \\
\mathbf{y}_{p}^{\prime}=\left\{y_{r p} \mid r=1, \ldots, s\right\},
\end{gathered}
$$

or (output-oriented target)

$$
\begin{gathered}
\mathbf{x}_{p}^{\prime}=\left\{x_{i p} \mid i=1, \ldots, m\right\}, \\
\mathbf{y}_{p}^{\prime}=\left\{\sum_{j \in R_{o}} y_{r p} \lambda_{j}^{*} \mid r=1, \ldots, s\right\} .
\end{gathered}
$$

Obviously, $\mathrm{DMU}_{p}^{\prime}$ corresponds to the ideal DMU having the same input/output isoquant as $\mathrm{DMU}_{p}$.

The reference-set $R_{o}$ for the six DMUs in Table 1 is shown in Table 3. If there are plural DMUs in the reference-set, the referred percentage is shown in the parentheses. Emptiness means being dim-efficient in the corresponding dimension. Table 3 tells us that, for example, DMU5, serves as the benchmark target for all other DMUs in Input 1 since it has the most excellent performance in the utilization of Input 1. Actually, DMU5 is the only unit getting a dim-efficiency score of unity in Input 1.

\section{An Application to 26 Supermarkets}

In this section, the proposed approaches are applied to 26 supermarkets located in the city of Nanjing, China. These supermarkets belong to the same chain and are therefore appropriate for internal comparison and benchmark learning. Two outputs are sales (unit: $10^{6}$ Yuan) and customer satisfaction, representing financial- and customer-based outcomes, respectively. Three inputs are shop size (unit: $10^{2} \mathrm{~m}^{2}$ ), promotion expenses (unit: \% of sales), and man-hour (unit: $10^{3} \mathrm{~h}$ ). Promotion expenses are limited to expenses related to store specific advertising or promotion, such as pointof-purchase displays or single store promotions. Man-hour refers to the labor force used within a certain period.

In this case, what decision makers care about is how supermarkets perform in a specific dimension. For instance, which one has the highest utilization rate of promotion expenses? How about the use of shop area in each super-

\begin{tabular}{|c|c|c|c|c|c|}
\hline ID & $\begin{array}{c}\text { Sales } \\
\left(10^{6}\right. \\
\text { Yuan }) \\
\end{array}$ & $\begin{array}{l}\text { Customer } \\
\text { satisfaction }\end{array}$ & $\begin{array}{c}\text { Shop } \\
\text { size } \\
\left(10^{2} \mathrm{~m}^{2}\right)\end{array}$ & $\begin{array}{c}\text { Promotion } \\
\text { (\% of } \\
\text { sales) }\end{array}$ & $\begin{array}{l}\text { Man-hour } \\
\quad\left(10^{3} \mathrm{~h}\right)\end{array}$ \\
\hline 1 & 3.7 & 3.6 & 7 & 2.1 & 32 \\
\hline 2 & 3.9 & 3.7 & 13 & 2.6 & 30 \\
\hline 3 & 3.6 & 3.7 & 13 & 2.9 & 28 \\
\hline 4 & 4.2 & 3.9 & 10 & 3.3 & 24 \\
\hline 5 & 4 & 3.9 & 12 & 3 & 38 \\
\hline 6 & 4 & 3.9 & 9 & 3.8 & 24 \\
\hline 7 & 3.2 & 4 & 9 & 2.2 & 32 \\
\hline 8 & 4.1 & 4 & 7 & 3.4 & 30 \\
\hline 9 & 3.6 & 4.1 & 7 & 3.5 & 24 \\
\hline 10 & 4.2 & 4.2 & 6 & 3.5 & 32 \\
\hline 11 & 4 & 4.2 & 7 & 3.5 & 28 \\
\hline 12 & 3.7 & 3.5 & 6 & 3 & 24 \\
\hline 13 & 4 & 3.6 & 6 & 2.5 & 25 \\
\hline 14 & 4.2 & 4.2 & 9 & 3.5 & 29 \\
\hline 15 & 4 & 4.3 & 10 & 4 & 26 \\
\hline 16 & 4.1 & 4 & 6 & 4 & 33 \\
\hline 17 & 4 & 4 & 11 & 3.5 & 25 \\
\hline 18 & 3.8 & 3.6 & 11 & 2.8 & 20 \\
\hline 19 & 4.6 & 4.3 & 12 & 4.2 & 30 \\
\hline 20 & 4.2 & 4.4 & 12 & 3.9 & 30 \\
\hline 21 & 4.4 & 4.3 & 6 & 3.8 & 32 \\
\hline 22 & 4.4 & 4.4 & 7 & 4.1 & 35 \\
\hline 23 & 4.5 & 4.5 & 12 & 5 & 33 \\
\hline 24 & 4.6 & 4.5 & 14 & 4.3 & 36 \\
\hline 25 & 4.6 & 4.5 & 7 & 3 & 35 \\
\hline 26 & 4.5 & 4.6 & 12 & 4.6 & 30 \\
\hline
\end{tabular}
market? How to enhance customer satisfaction? However, traditional DEA models can only evaluate performances from
TABLE 4: Input-output data for 26 supermarkets.

an overall perspective. Approaches proposed in this paper can provide more information and are suitable for such requirements.

Data set collected in a season for the 26 supermarkets is as shown in Table 4.

Table 5 reports CCR efficiency scores from model (2) in the second column and dim-efficiency scores calculated by Approaches 1 and 2 in the third to the last columns. Rows in bold type are CCR DEA efficient units. From Table 5 we can see that no supermarket performs best in all dimensions. Each of them has weak points in certain aspects. For example, supermarket 1 is good at sales, satisfying customer and promotion, but performs not so well in the utilization of shop area and labor force. Table 5 also tells us that dimefficiency scores derived by Approach 1 or 2 are no larger than their corresponding CCR efficiency scores. This property can be deduced from their definitions. Another phenomenon in Table 5 that we can find is that dim-efficiency scores derived by Approach 2 are no less than those by Approach 1; for example, Figure 2 depicts the dim-efficiency scores of supermarket 2 by the two approaches. But it is not always the case, since there is no direct relationship between the two 
TABLE 5: CCR efficiency and dim-efficiency scores.

\begin{tabular}{|c|c|c|c|c|c|c|c|c|c|c|c|}
\hline \multirow{2}{*}{ ID } & \multirow{2}{*}{ CCR } & \multicolumn{2}{|c|}{ Sales } & \multicolumn{2}{|c|}{ Customer satisfaction } & \multicolumn{2}{|c|}{ Shop size } & \multicolumn{2}{|c|}{ Promotion } & \multicolumn{2}{|c|}{ Man-hour } \\
\hline & & $\mathrm{Al}^{*}$ & $\mathrm{~A} 2^{\dagger}$ & $\mathrm{A} 1$ & A2 & $\mathrm{A} 1$ & A2 & $\mathrm{A} 1$ & A2 & $\mathrm{A} 1$ & A2 \\
\hline 1 & 1 & 1 & 1 & 1 & 1 & 0.426 & 0.721 & 1 & 1 & 0.399 & 0.625 \\
\hline 2 & 0.927 & 0.354 & 0.910 & 0.627 & 0.888 & 0 & 0.409 & 0.624 & 0.851 & 0.324 & 0.685 \\
\hline 3 & 0.874 & 0.174 & 0.791 & 0.720 & 0.870 & 0.003 & 0.397 & 0.526 & 0.734 & 0.357 & 0.734 \\
\hline 4 & 0.988 & 0.988 & 0.988 & 0.381 & 0.943 & 0.25 & 0.573 & 0.002 & 0.722 & 0.764 & 0.921 \\
\hline 5 & 0.813 & 0.285 & 0.792 & 0.545 & 0.773 & 0.002 & 0.455 & 0.527 & 0.758 & 0.293 & 0.570 \\
\hline 6 & 0.985 & 0.594 & 0.964 & 0.415 & 0.935 & 0.233 & 0.606 & 0.004 & 0.598 & 0.756 & 0.903 \\
\hline 7 & 1 & 0.282 & 0.835 & 1 & 1 & 0.344 & 0.620 & 1 & 1 & 0.545 & 0.694 \\
\hline 8 & 0.915 & 0.059 & 0.870 & 0.914 & 0.915 & 0.416 & 0.799 & 0.201 & 0.686 & 0.351 & 0.741 \\
\hline 9 & 1 & 0.549 & 0.909 & 1 & 1 & 0.598 & 0.817 & 0.454 & 0.658 & 0.939 & 0.949 \\
\hline 10 & 1 & 0.084 & 0.975 & 1 & 1 & 0.722 & 0.977 & 0.308 & 0.695 & 0.127 & 0.729 \\
\hline 11 & 0.977 & 0.034 & 0.887 & 0.976 & 0.977 & 0.45 & 0.837 & 0.222 & 0.687 & 0.341 & 0.833 \\
\hline 12 & 0.977 & 0.585 & 0.958 & 0.630 & 0.950 & 0.436 & 0.841 & 0.006 & 0.700 & 0.811 & 0.811 \\
\hline 13 & 1 & 1 & 1 & 1 & 1 & 0.808 & 0.909 & 0.807 & 0.908 & 0.855 & 0.842 \\
\hline 14 & 0.915 & 0.025 & 0.868 & 0.914 & 0.915 & 0.09 & 0.651 & 0.377 & 0.695 & 0.471 & 0.805 \\
\hline 15 & 0.95 & 0.052 & 0.884 & 0.949 & 0.950 & 0.089 & 0.6 & 0.011 & 0.612 & 0.878 & 0.919 \\
\hline 16 & 0.932 & 0.931 & 0.932 & 0.480 & 0.930 & 0.932 & 0.932 & 0.017 & 0.583 & 0.027 & 0.673 \\
\hline 17 & 0.918 & 0.535 & 0.892 & 0.400 & 0.916 & 0.677 & 0.507 & 0.011 & 0.662 & 0.677 & 0.889 \\
\hline 18 & 1 & 1 & 1 & 1 & 1 & 0.31 & 0.471 & 0.669 & 0.770 & 1 & 1 \\
\hline 19 & 0.877 & 0.626 & 0.87 & 0.362 & 0.830 & 0.218 & 0.523 & 0.007 & 0.622 & 0.667 & 0.807 \\
\hline 20 & 0.875 & 0.004 & 0.798 & 0.875 & 0.876 & 0.101 & 0.512 & 0.366 & 0.646 & 0.418 & 0.815 \\
\hline 21 & 1 & 1 & 1 & 1 & 1 & 1 & 1 & 0.321 & 0.659 & 0.627 & 0.747 \\
\hline 22 & 0.915 & 0.024 & 0.880 & 0.914 & 0.915 & 0.386 & 0.877 & 0.222 & 0.622 & 0.349 & 0.698 \\
\hline 23 & 0.820 & 0.478 & 0.793 & 0.345 & 0.786 & 0.189 & 0.523 & 0.001 & 0.521 & 0.632 & 0.758 \\
\hline 24 & 0.774 & 0.007 & 0.733 & 0.774 & 0.775 & 0.09 & 0.449 & 0.376 & 0.610 & 0.384 & 0.694 \\
\hline 25 & 1 & 0.739 & 0.980 & 1 & 1 & 0.759 & 0.897 & 0.667 & 0.874 & 0.369 & 0.714 \\
\hline 26 & 0.890 & 0.383 & 0.855 & 0.514 & 0.878 & 0.22 & 0.535 & 0.002 & 0.576 & 0.672 & 0.852 \\
\hline
\end{tabular}

${ }^{*}$ A1 stands for dim-efficiency score derived by Approach 1.

${ }^{\dagger} \mathrm{A} 2$ stands for dim-efficiency score derived by Approach 2 .

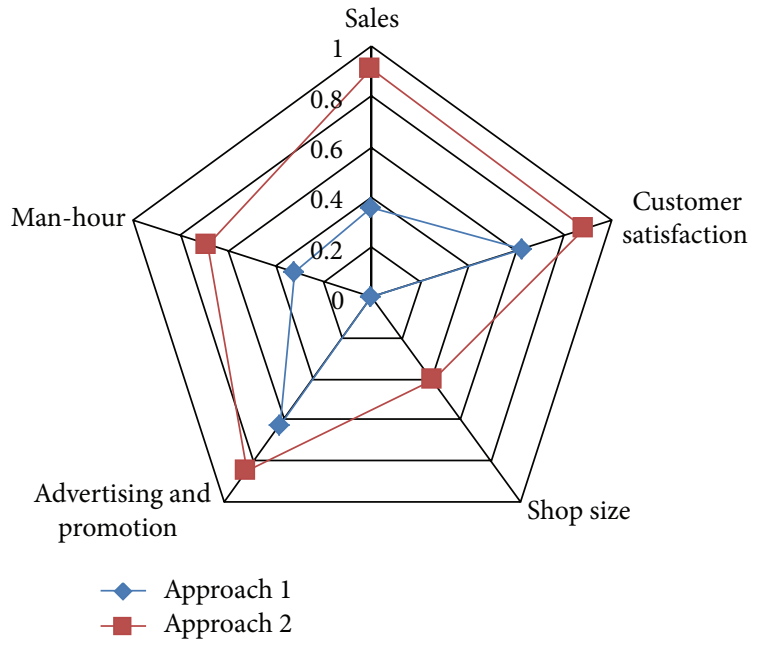

FIGURE 2: Dim-efficiency scores of supermarket 2 by the two approaches.

approaches by definition. One thing they have in common is that the resulting classification of dim-efficient and diminefficient DMUs is the same. For instance, in the dimension of sales, $\{1,13,18,21\}$ fall into the dim-efficient category and others into the dim-inefficient category, no matter which approach you choose.

Reference-sets for dim-inefficient units obtained by Approach 2 are shown in Table 6. If there are plural DMUs in the reference-set, the referred percentage is shown in the parentheses. In the dimension of sales, $\{1,13,18,21\}$ are dimefficient supermarkets and therefore should serve as role models on how to increase sales. Although supermarket 1 is evaluated to be CCR DEA efficient, it can also learn from supermarket 21 about the utilization of shop area and from supermarket 18 about the use of labor force, whereas supermarkets 18 and 21 can learn from supermarket 1 about the efficient utilization of advertising and promotion expenses. With the assistance of the proposed approaches, all DMUs can learn from each other in different dimensions and come to a mutual learning situation.

\section{Concluding Remarks}

This paper proposes two approaches to measure the efficiency of DMUs in each dimension. One measures efficiency in a multiplier-model form by further processing the original multiplier CCR model, while the other calculates efficiency in envelopment-model form by comparing with an ideal 
TABLE 6: Reference-sets as benchmark targets by Approach 2.

\begin{tabular}{|c|c|c|c|c|c|}
\hline ID & Sales & Customer satisfaction & Shop size & Promotion & Man-hour \\
\hline 1 & $\mathrm{DE}^{*}$ & $\mathrm{DE}$ & 21 & $\mathrm{DE}$ & 18 \\
\hline 2 & $1(31 \%), 13(69 \%)$ & $7(67 \%), 18(33 \%)$ & 21 & 1 & 18 \\
\hline 3 & $13(90 \%), 18(10 \%)$ & $7(42 \%), 18(58 \%)$ & 21 & $1(77 \%), 7(23 \%)$ & 18 \\
\hline 4 & $13(39 \%), 18(61 \%)$ & $9(37 \%), 13(10 \%), 18(53 \%)$ & 21 & 1 & 18 \\
\hline 5 & $1(53 \%), 13(47 \%)$ & 7 (79\%), 13 (3\%), 18 (18\%) & 21 & $1(99 \%), 7(1 \%)$ & 18 \\
\hline 6 & $13(52 \%), 18(48 \%)$ & $9(67 \%), 18(33 \%)$ & 21 & $1(99 \%), 7(1 \%)$ & 18 \\
\hline 7 & $1(88 \%), 13(12 \%)$ & $\mathrm{DE}$ & 21 & $\mathrm{DE}$ & 18 \\
\hline 8 & $13(89 \%), 21(11 \%)$ & $9(20 \%), 13(53 \%), 21(27 \%)$ & 21 & $1(99 \%), 7(1 \%)$ & 18 \\
\hline 9 & 13 (81\%), 18 (19\%) & $\mathrm{DE}$ & 21 & $1(36 \%), 7(64 \%)$ & 18 \\
\hline 10 & $13(21 \%), 21(79 \%)$ & $\mathrm{DE}$ & 21 & $1(88 \%), 7(12 \%)$ & 18 \\
\hline 11 & $13(96 \%), 18(4 \%)$ & 9 (49\%), $13(29 \%), 21(22 \%)$ & 21 & $1(68 \%), 7(32 \%)$ & 18 \\
\hline 12 & $13(96 \%), 18(4 \%)$ & 9 (49\%), $13(29 \%), 21(22 \%)$ & 21 & 1 & 18 \\
\hline 13 & $\mathrm{DE}$ & $\mathrm{DE}$ & 21 & 1 & 18 \\
\hline 14 & $13(74 \%), 18(26 \%)$ & $9(32 \%), 13(48 \%), 18(20 \%)$ & 21 & $1(88 \%), 7(12 \%)$ & 18 \\
\hline 15 & $13(49 \%), 18(51 \%)$ & $9(63 \%), 18(37 \%)$ & 21 & $1(59 \%), 7(41 \%)$ & 18 \\
\hline 16 & 21 & 21 & 21 & $1(99 \%), 7(1 \%)$ & 18 \\
\hline 17 & $13(32 \%), 18(68 \%)$ & $9(35 \%), 13(4 \%), 18(60 \%)$ & 21 & $1(88 \%), 7(12 \%)$ & 18 \\
\hline 18 & $\mathrm{DE}$ & $\mathrm{DE}$ & 21 & 1 & $\mathrm{DE}$ \\
\hline 19 & $13(44 \%), 18(56 \%)$ & $9(49 \%), 13(6 \%), 18(45 \%)$ & 21 & 1 & 18 \\
\hline 20 & $13(44 \%), 18(56 \%)$ & $9(24 \%), 13(24 \%), 18(51 \%)$ & 21 & $1(69 \%), 7(31 \%)$ & 18 \\
\hline 21 & $\mathrm{DE}$ & $\mathrm{DE}$ & $\mathrm{DE}$ & $1(98 \%), 7(2 \%)$ & 18 \\
\hline 22 & $13(27 \%), 21(73 \%)$ & $9(6 \%), 13(16 \%), 21(79 \%)$ & 21 & $1(88 \%), 7(12 \%)$ & 18 \\
\hline 23 & 13 (56\%), $18(44 \%)$ & $9(71 \%), 18(29 \%)$ & 21 & $1(88 \%), 7(12 \%)$ & 18 \\
\hline 24 & $13(48 \%), 18(52 \%)$ & $9(1 \%), 13(46 \%), 18(53 \%)$ & 21 & $1(98 \%), 7(2 \%)$ & 18 \\
\hline 25 & $13(94 \%), 21(6 \%)$ & $\mathrm{DE}$ & 21 & $1(98 \%), 7(2 \%)$ & 18 \\
\hline 26 & $13(44 \%), 18(56 \%)$ & $9(57 \%), 18(43 \%)$ & 21 & $1(79 \%), 7(21 \%)$ & 18 \\
\hline
\end{tabular}

${ }^{*} \mathrm{DE}$ means that the DMU is dim-efficient and does not need to select benchmark targets.

DMU constructed under a given input/output isoquant. Benchmarking advices are provided by Approach 2 for diminefficient DMUs to make improvement in the associated dimension.

In the models of Approach 1, all dimensions are considered, whereas in Approach 2 only the dimension under evaluation is taken into account. Approach 2 is especially applicable in the case when input/output factors cannot be substituted for each other [19].

\section{Conflict of Interests}

The authors declare that there is no conflict of interests regarding the publication of this paper.

\section{Acknowledgment}

This work was supported by the National Natural Science Foundation of China (70971137).

\section{References}

[1] A. Charnes, W. W. Cooper, and E. Rhodes, "Measuring the efficiency of decision making units," European Journal of Operational Research, vol. 2, no. 6, pp. 429-444, 1978.
[2] W. W. Cooper, L. M. Seiford, and J. Zhu, Handbook on Data Envelopment Analysis, Springer, New York, NY, USA, 2011.

[3] S. Lim, H. Bae, and L. H. Lee, "A study on the selection of benchmarking paths in DEA," Expert Systems with Applications, vol. 38, no. 6, pp. 7665-7673, 2011.

[4] X. Yang and H. Morita, "Efficiency improvement from multiple perspectives: an application to Japanese banking industry," Omega, vol. 41, no. 3, pp. 501-509, 2013.

[5] F. Aleskerov, H. Ersel, and R. Yolalan, "Multicriterial ranking approach for evaluating bank branch performance," International Journal of Information Technology \& Decision Making, vol. 3, no. 2, pp. 321-335, 2004.

[6] S. Speelman, M. D’Haese, J. Buysse, and L. D’Haese, “A measure for the efficiency of water use and its determinants, a case study of small-scale irrigation schemes in North-West Province, South Africa," Agricultural Systems, vol. 98, no. 1, pp. 31-39, 2008.

[7] L. D. Taminia, B. Larue, and G. West, “Technical and environmental efficiencies and best management practices in agriculture," Applied Economics, vol. 44, no. 13, pp. 1659-1672, 2012.

[8] B. Hollingsworth, "The measurement of efficiency and productivity of health care delivery," Health Economics, vol. 17, no. 10, pp. 1107-1128, 2008.

[9] S. Peck, "Health care benchmarking and performance evaluation: an assessment using data envelopment healthcare benchmarking and performance evaluation: analysis (DEA)," Journal of the Operational Research Society, vol. 60, no. 9, pp. 1302-1302, 2009. 
[10] S. G. Barbosa and V. E. Wilhelm, "Evaluation of the performance of public schools through data envelopment analysis," Acta Scientiarum-Technology, vol. 31, no. 1, pp. 71-79, 2009.

[11] M. C. Portela, A. S. Camanho, and A. Keshvari, "Assessing the evolution of school performance and value-added: trends over four years," Journal of Productivity Analysis, vol. 39, no. 1, pp. 1-14, 2013.

[12] S. Lozano, E. Gutiérrez, and P. Moreno, "Network DEA approach to airports performance assessment considering undesirable outputs," Applied Mathematical Modelling, vol. 37, no. 4, pp. 1665-1676, 2013.

[13] S. Lozano and E. Gutiérrez, "Slacks-based measure of efficiency of airports with airplanes delays as undesirable outputs," Computers \& Operations Research, vol. 38, no. 1, pp. 131-139, 2011.

[14] H. K. Hong, S. H. Ha, C. K. Shin, S. C. Park, and S. H. Kim, "Evaluating the efficiency of system integration projects using data envelopment analysis (DEA) and machine learning," Expert Systems with Applications, vol. 16, no. 3, pp. 283-296, 1999.

[15] W.-M. Lu and M.-H. Chen, "A benchmark-learning roadmap for the Military Finance Center," Mathematical and Computer Modelling, vol. 53, no. 9-10, pp. 1833-1843, 2011.

[16] J. M. Wagner and D. G. Shimshak, "Stepwise selection of variables in data envelopment analysis: procedures and managerial perspectives," European Journal of Operational Research, vol. 180, no. 1, pp. 57-67, 2007.

[17] J. Sun, J. Wu, and D. Guo, "Performance ranking of units considering ideal and anti-ideal DMU with common weights," Applied Mathematical Modelling, vol. 37, no. 9, pp. 6301-6310, 2013.

[18] K. Tone, "A slacks-based measure of efficiency in data envelopment analysis," European Journal of Operational Research, vol. 130, no. 3, pp. 498-509, 2001.

[19] C. Tofallis, "Input efficiency profiling: an application to airlines," Computers \& Operations Research, vol. 24, no. 3, pp. 253-258, 1997. 


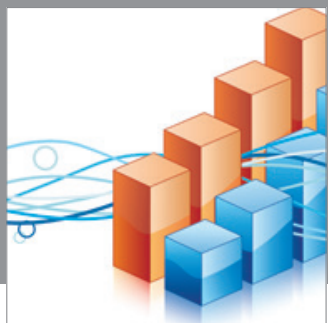

Advances in

Operations Research

mansans

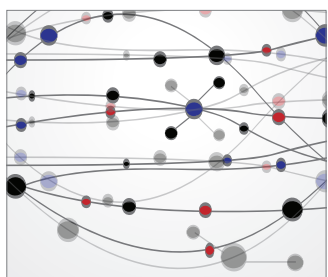

The Scientific World Journal
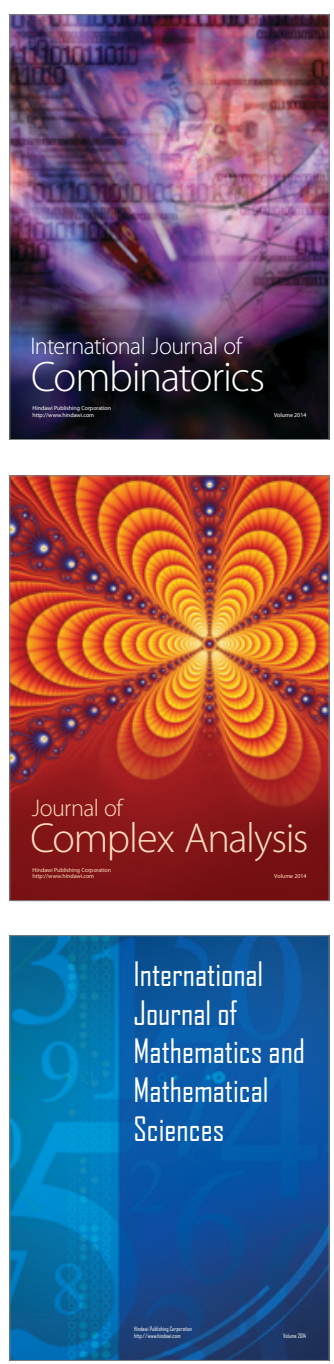
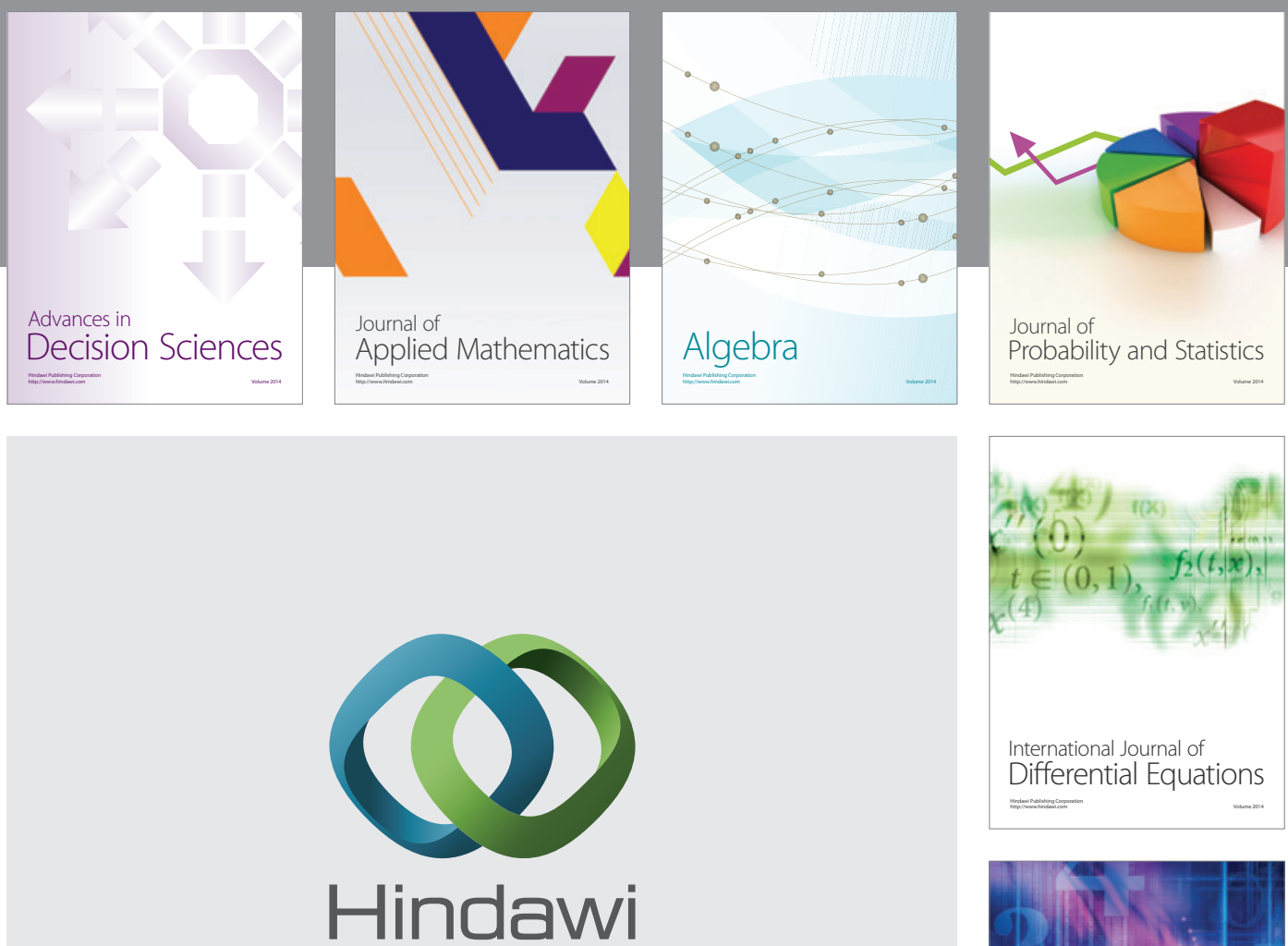

Submit your manuscripts at http://www.hindawi.com
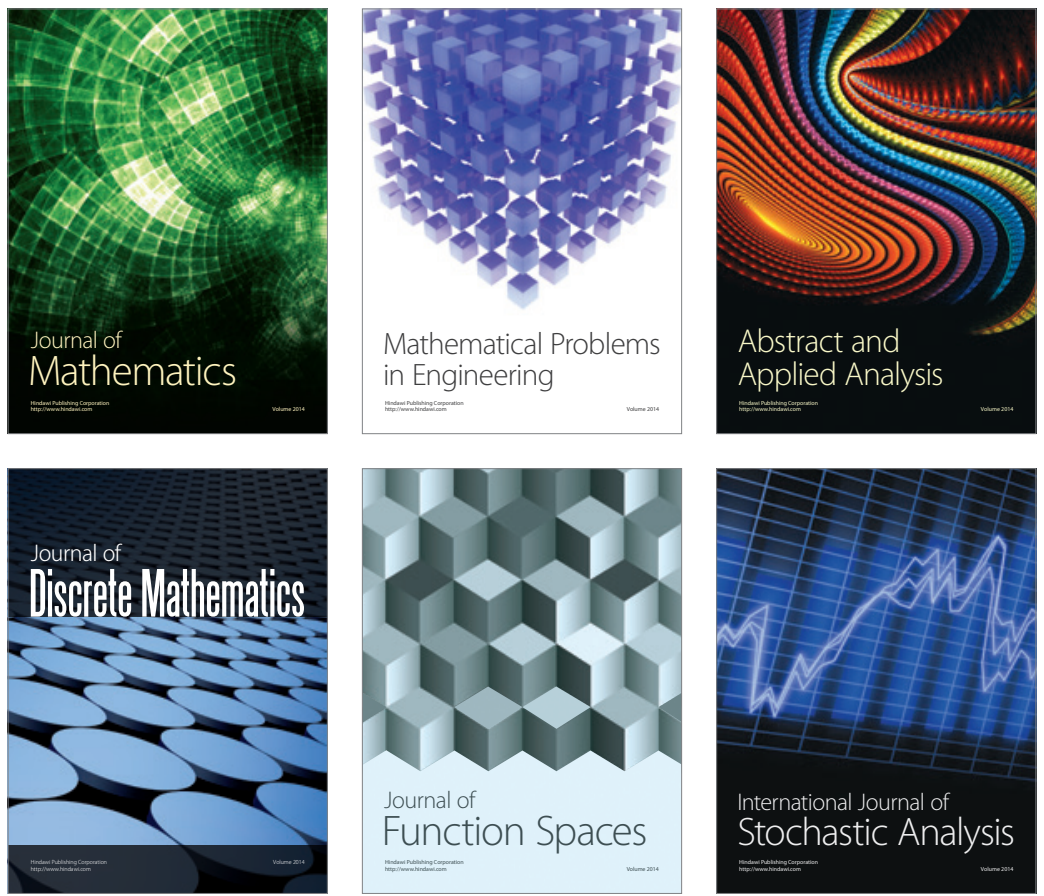

Journal of

Function Spaces

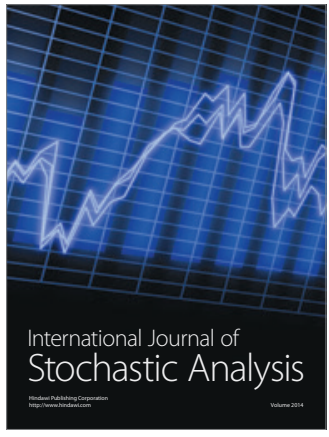

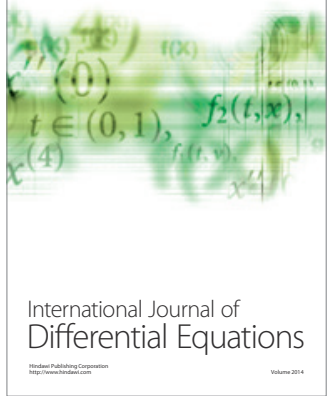
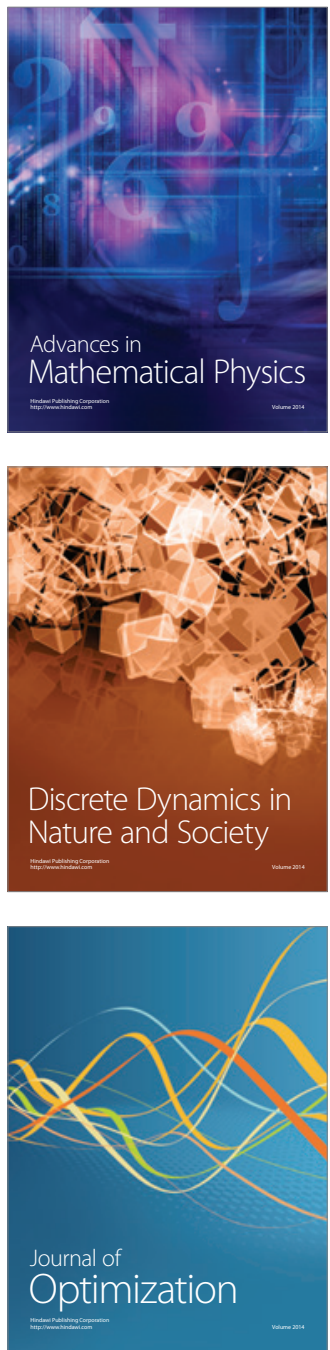